\title{
Laterality of pain in migraine distinguished by interictal rates of habituation of electrodermal responses to visual and auditory stimuli
}

\author{
J H GRUZELIER, T NICOLAOU, J F CONNOLLY, R C PEATFIELD, \\ P T G DAVIES, F CLIFFORD-ROSE
}

\begin{abstract}
From the Laboratory of Neuro-Psychophysiology, Department of Psychiatry and Academic Unit of Neuroscience, Charing Cross and Westminster Medical School and The Princess Margaret Migraine Clinic, Charing Cross Hospital, London, UK
\end{abstract}

SUMMARY Support is provided for a primary neural factor in migraine by studies in autonomic responsiveness to sensory stimuli in relation to the laterality of pain. Migraineurs with consistently lateralised headaches were found in two studies to exhibit extremes of autonomic responsiveness to sensory stimuli during the interictal phase. The direction of responsiveness was predictive of the laterality of pain; left-sided pain was associated with under-responsiveness and fast habituation, right-sided pain with over-responsiveness and slow habituation. Bipolarity in rate of habituation is consistent with a defect in a regulatory mechanism that controls regional cerebral activation such as the diffuse thalamic projection system.

While in the last century consideration was given to the primacy of neural influences in migraine, ${ }^{1}$ until recently most research has sought an explanation through changes in the extra-cranial vasculature, notably dilatation of the cephalic artery found to coincide with the experience of throbbing pain. ${ }^{2}$ Currently there is a renewal of interest in the neural hypothesis, ${ }^{3-5}$ largely due to changes of regional cerebral blood flow during migraine attacks whose observation has been made possible through advances in brain imaging. ${ }^{6-8}$ Techniques involving inhalation or carotid injection of xenon 133 have revealed regional abnormalities, often lasting for days, as well as a shorter lasting focal oligaemia spreading anteriorly from the occipital lobe to primary sensory motor cortex. Focal neurological symptoms including scintillations, paraesthesia, and aphasia did not coincide with the oligaemia. ${ }^{78}$

A neural explanation is compatible with other features of migraine. A range of precipitating factors has been documented which reflects changes in the external and internal environment.These are as diverse as

Address for reprint requests: Dr John Gruzelier, Department of Psychiatry, Charing Cross and Westminster Medical School, Fulham Palace Road, London W6 8RF, UK.

Received 20 May 1986 and in revised form 28 July 1986. Accepted 1 August 1986 bright light, noise, sharp smells, climatic changes, stress, mental and physical exhaustion, sleep, relaxation, food, alcohol, pharmaceutical preparations, menstruation, fluid retention and autonomic instability. ${ }^{9}$ Electrophysiological recordings of brain electrical activity in the interictal phase have shown delayed recovery both from photic driving and hyperventilation. ${ }^{10}$ In our laboratory cortical evoked potentials (EPs) to a reversing checkerboard showed delayed latencies in components between $100-200 \mathrm{~ms}^{11}$ This is a range in which central nonsensory processes such as attention are reflected in the evoked potential. ${ }^{12}$ EP amplitudes were also sometimes enlarged within this range, a feature found to be dependent on whether the patient consistently localised the headache to the left side. We confirmed the importance of central regulatory influences on perception in a second EP experiment with unpatterned light flashes of variable intensity. ${ }^{13}$

Here the importance of central regulatory processes in migraine have been extended by examining the effects of sensory stimuli on phasic autonomic responses. Autonomic dysregulation has been suggested as a predispositional factor in migraine. ${ }^{14} \mathrm{We}$ measured the Pavlovian orienting response and its habituation with stimulus repetition. ${ }^{15}$ The orienting response reflects investment of attention in a novel stimulus and is accompanied by electrocortical desynchronisation and phasic autonomic responses in 
addition to behavioural signs of orientation. Processes of orienting and habituation involve the nonspecific thalamo-cortical activation system as well as limbic regulatory mechanisms. ${ }^{15-17}$ We examined the electrodermal response because, by virtue of its sole sympathetic mediation, it is a particularly clear indicator of orienting and habituation processes.

As in our previous studies migraineurs were selected whose experience of pain was consistently lateralised. Cerebral asymmetry has been found to relate to rate of habituation and degree of reactivity in the electrodermal system, fast habituation being associated with the left hemisphere, and slow habituation with the right. ${ }^{1819}$ If the laterality of pain in migraine should coincide with differences in rate of habituation in patients free of headache at the time, this would give added credence to the neural hypothesis. Furthermore it would suggest an intra-cerebral genesis for the pain of migraine. Two experiments were conducted. In the first a series of unpatterned flashes acted as the orienting stimuli and normal controls were compared with patients having left or rightsided migraine or bilateral tension headache. In a replication study auditory stimuli were used with left and right-sided migraineurs.

\section{Subjects}

In Experiment I 30 female subjects were tested: five with left-sided migraine, six with right-sided migraine, seven with bilateral tension headache, and 12 medically fit controls. Headache was the only major disability in patients and was diagnosed according to criteria proposed by the Ad Hoc Committee. ${ }^{20}$ All migraine cases had nausea or visual symptoms or both; there were no group differences in associated symptoms such as photophobia, scintillation, dysphasia, etc. Subjects were dextral according to a modified Oldfield scale, with the exception of one patient with left-sided migraine, two with tension headache and one control. Ages ranged from 15 to 50 years and did not distinguish the groups. Nor did the groups differ in ages of first onset of headache which ranged between 6 and 25 years: left migraine $X 15.2$ years; right migraine $X 14.5$ years; tension headache $X \quad 17.7$ years. There were no group differences in the frequency of attacks which on average occurred once a week. All the migraine patients had classical migraine except one left-sided case with common migraine. In case group differences could be attributed to anxiety, subjects filled out the IPAT Anxiety Scale Questionnaire; ${ }^{21}$ there were no group differences $(F=$ 0.41 , df $=2.27$, NS). Patients were all examined in the interictal period. Prophylactic medication was taken by one patient with left-sided migraine, four with right-sided migraine and three with tension headache. Two patients were on propranolol, one in each migraine group. Otherwise the right-sided patients were on flunarazine, pizotifen and clonidine respectively, and the tension headache cases on diazepam, clonidine and chlorpromazine.

In Experiment II the patients were seven left-sided and six right-sided migraineurs, with one male in each group. All patients were unmedicated; none had been receiving prophylactic medication in the preceding month and symptomatic medication had not been used for 4 days. All experienced unilateral pain in $90 \%$ or more of episodes. Right-sided patients were on average younger, though the groups did not differ statistically; X 39 years compared with X 44 years for left-sided patients, $(t=1 \cdot 1, N S)$. All patients were dextral as assessed by a modified Oldfield questionnaire. The groups did not differ in the frequency of headache (on average one a week), nor did they differ in age of first onset (X 24 years). There was no requirement that the patients experienced particular sensory symptoms. Three left-sided and two rightsided patients had common migraine. Two patients in each group had a family history of headache. One left-sided patient experienced hemianopia, one right-sided case dysphasia, while five of the right-sided cases reported nausea and vomiting as did four of the left-sided cases. None of the patients experienced unilateral weakness or other somatosensory symptoms. On the IPAT Anxiety Scale there was a tendency for left-sided patients to experience more anxiety $(t$ $=2.03, \mathrm{p}<0.07$ ); X 34.17 right-sided, X 49.83 left-sided. The direction of the difference indicated that any autonomic over-responsiveness in right-sided patients could not be attributed to higher anxiety levels. Patients had on average been free of headaches for $\mathbf{8}$ days with a minimum of 5 days; there was no group difference.

\section{Procedure}

In Experiment I the stimuli consisted of 20 flashes of white light with an intensity of 10 foot lamberts (107.6 lux), a level that would not elicit a defence reaction..$^{22}$ The interstimulus intervals varied between 10 and 35 seconds. Midway through the 30 second interval between the 10th and 11th flashes an orange light was presented to serve as a dishabituating stimulus. The flashes were produced by two Kodak Carousel S-AV 1000 projectors modified to give shutter rise and fall times of 1.5 seconds, and back-projected onto an opaque window. The stimuli subtended angles of $20^{\circ}$ horizontally and $16^{\circ}$ vertically and were viewed in a dimly lit, sound-attenuated room. The auditory stimuli were 21 1-second tones of $1000 \mathrm{~Hz}$, with graded rise and decay times, produced by a Sony TESD cassette recorder and presented through headphones (Koss type 6LC). The interstimulus intervals were between 20 and 40 seconds.

Skin conductance was measured between silver-silver chloride cup electrodes, $1 \mathrm{~cm}$ in diameter, fastened through a $\mathrm{KCl}$ electrolyte to the middle phalanx of the first and second fingers of each hand, and connected to a Grass model 7D polygraph via a constant voltage skin conductance system. The orienting response was defined as occurring between 1 and 3 seconds after flash onset. Nonspecific responses were defined as those occurring outside these intervals. The minimum gain was $0.02 \mu \mathrm{mho} / \mathrm{cm}$ with a minimum amplitude criterion of $1 \mathrm{~mm}$. Habituation was said to occur when there was an absence of responses on two successive trials. Skin conductance levels were recorded at intervals during each sequence. A tonic change in skin conductance was defined as the difference between the highest and lowest levels during the session. In Experiment II finger skin temperature, recorded as a safeguard against abnormally low temperature which might attenuate electrodermal responses, was mea- 
Table 1 Left and right hand means and standard deviations for the skin conductance measures

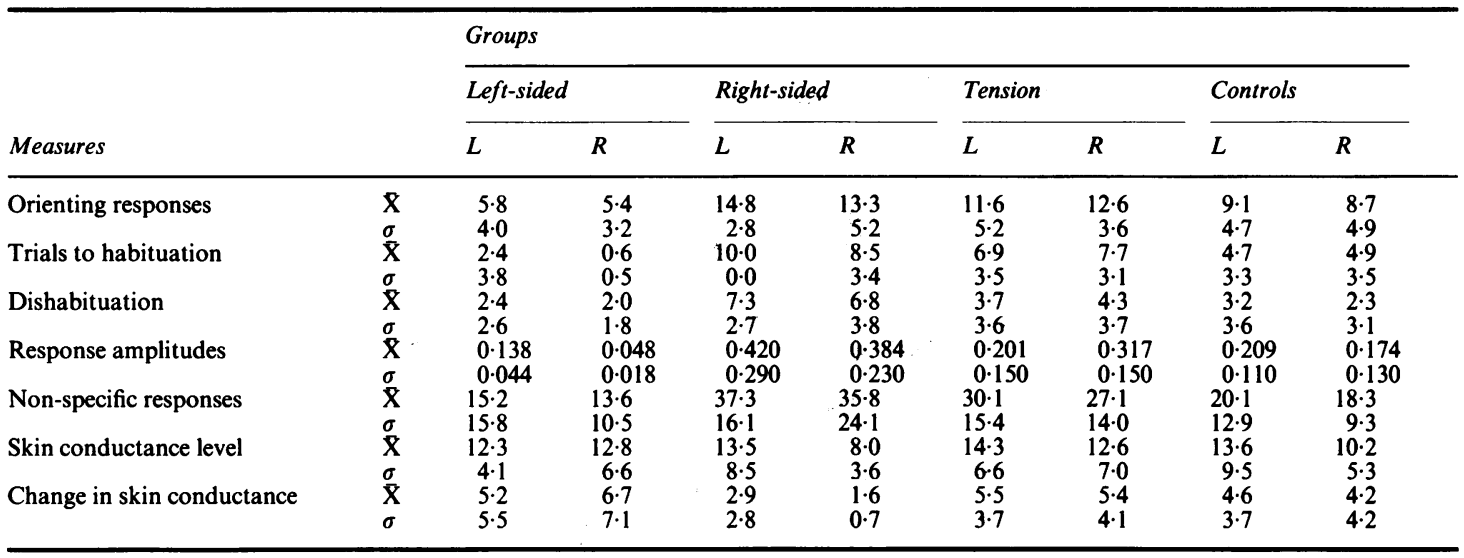

Table 2 Levels of statistical significance for group comparisons of the skin conductance measures (Comparisons are also provided for the left-sided migraineurs with the sinistral patient excluded $(N=4)$

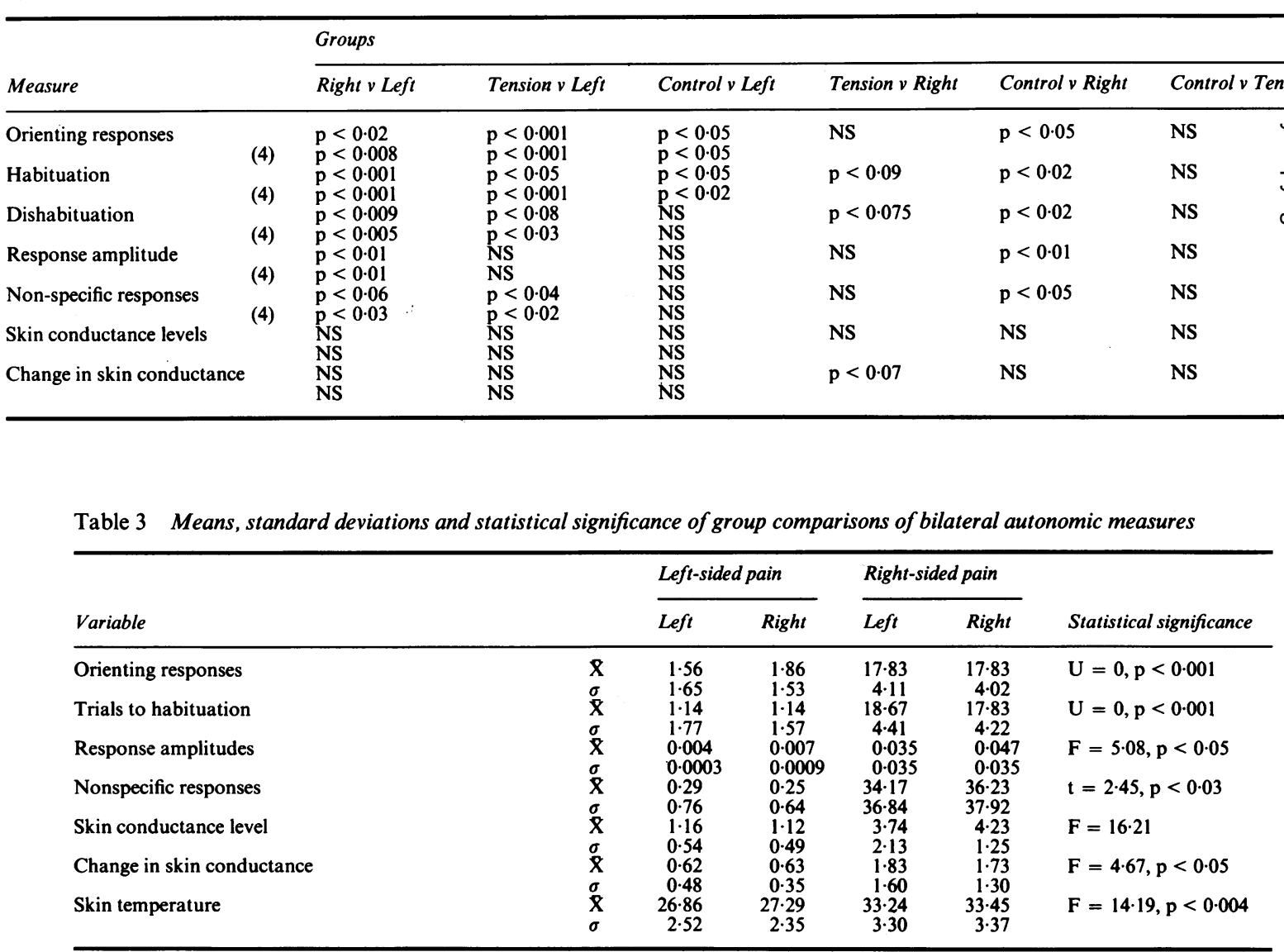




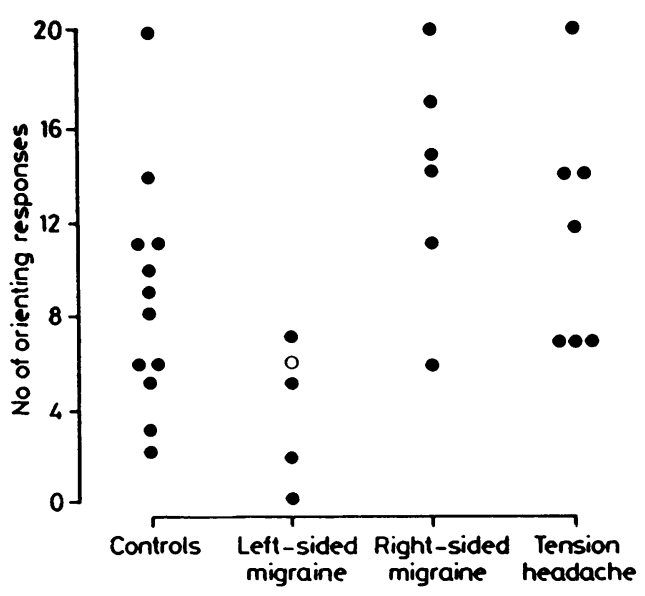

Fig 1 Number of responses to the flashes for each subject in the four groups. The unfilled circle indicates the left-handed migraineur.

sured with a Comark digital thermometer with a thermistor held between thumb and forefinger. None of the patients had temperatures that were sufficiently low to reduce sweating (table 3 ). The test procedure was explained as a measure of the patient's relaxation: occasional flashes would be presented to the screen in front of them which they were asked to watch in a relaxed and passive frame of mind until the experimenter re-entered the room (Experiment I). In the second experiment occasional sounds would be heard through headphones which they were to listen to in a relaxed and passive frame of mind.

\section{Results}

\section{Experiment I}

The main finding was a striking separation between left and right-sided migraineurs in responsiveness and habituation to the flashes. Figure 1 shows the number of orienting responses and fig 2 the number of trials until habituation before the dishabituating stimulus. Right-sided cases were highly responsive, more so than the patients with tension headache and the healthy controls, while the left-sided migraineurs were less responsive than all other groups. In fact there was no overlap in rate of habituation between the migraineurs divided by laterality of pain; the one left-hander was midway between the group in habituation, a result in keeping with evidence of more bilateral representation of psychological processes in sinistrals. ${ }^{23}$ The direction of bipolarity in responsiveness was compatible with evidence of hemispheric influences on habituation, that is, fast habituation with the left hemisphere and slow habituation with the right hemisphere. ${ }^{19}$

Means and standard deviations of the elec- trodermal variables are shown in table 1 and levels of statistical significance for group comparisons using the Mann Whitney U Test or Student $t$ test as appropriate in table 2. The differences in responsiveness $(p<0.02)$ and habituation $(p<0.001)$ between the migraineurs found parallels in the amplitudes of responses and rates of dishabituation $(p<0.009)$. In all aspects of the phasic orienting response the migraineur groups differed significantly from one another, whether or not the left-handed migraineur was excluded. In contrast, in measures of non-specific or tonic reactivity differences between left and rightsided migraineurs were either absent or less reliable. This was true of non-specific responses, levels of skin conductance, and changes in skin conductance such as may accompany tonic shifts in activation. Thus migraineurs were distinguished by phasic stimulus evoked responsiveness, suggesting a stimulusresponse mechanism rather than longer lasting tonic changes or non-specific reactivity.

From tables 1 and 2 it can be seen that compared with normal controls migraineurs were at both extremes of phasic responsiveness; with left-sided patients having fewer orienting responses and faster habituation $(p<0.05)$ and right-sided patients having higher levels of phasic responsiveness $(p<0.05)$ than controls. Patients with tension headache were not distinguished from normal controls, though on average patients were more responsive. The tendency in tension headache towards above normal responsiveness precluded any clear cut differences from right-sided migraineurs, but accentuated differences from left-sided migraineurs $(p<0.001)$. There was a tendency $(p<0.07)$ for right-sided migraineurs to have smaller changes in tonic skin conductance than patients with tension headache, drawing attention to

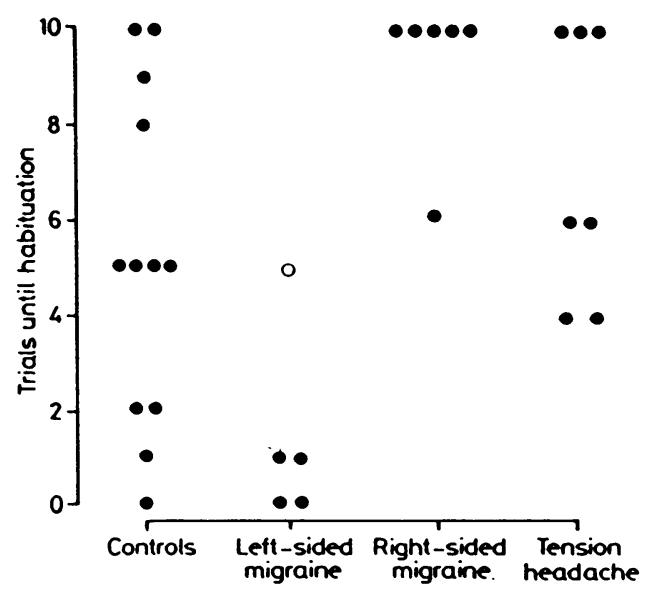

Fig 2 Number of trials until habituation to the flashes before the dishabituating stimulus on trial ten. 


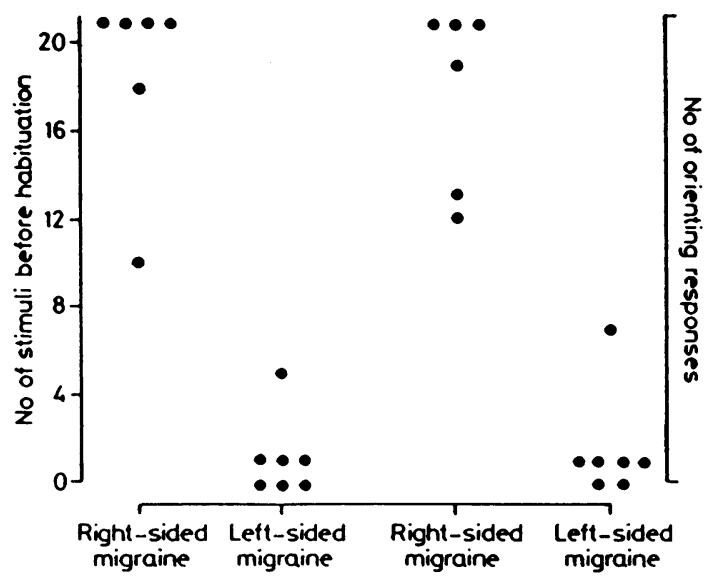

Fig 3 Number of trials until habituation and number of orienting responses to auditory stimuli.

the fact that in general phasic and tonic responses bore no close relationship.

Lateral differences in skin conductance activity were assessed with the conventional laterality index (left minus right, divided by left plus right). The groups were ranked as follows: left-sided migraineurs $0 \cdot 455$, controls $0 \cdot 151$, tension headaches $0 \cdot 144$, rightsided migraineurs $0 \cdot 128$. Student $t$ tests showed that the migraineurs differed significantly $(t=2 \cdot 62, \mathrm{p}<$ 0.03 ), with the left-sided migraineurs showing the larger asymmetry in the direction previously found to accompany dominance of left hemispheric influences. ${ }^{1819}$

In summary, the direction of sympathetic responsiveness was predictive of the laterality of pain in migraine. Underactivity was associated with leftsided pain and overactivity with right-sided pain. As four of the patients were on prophylactic medication a replication was planned with unmedicated patients. Auditory rather than visual stimuli were chosen to test the involvement of a generalised corticalautonomic regulatory mechanism.

\section{Experiment II}

The number of orienting responses to the 21 tonies, as well as the number of tones presented before habituation, are shown in fig 3. The bimodality in responsiveness to visual stimuli in the first experiment was replicated with auditory stimuli. Right-sided cases were highly responsive with $4 / 6$ patients failing to habituate, three of whom gave the maximum number of responses. The majority of left-sided cases were under-responsive with $6 / 7$ giving one response or none. There was no overlap between the groups in either rate of habituation or number of responses. In this experiment the groups also differed on all other autonomic measures (see table 3). The migraineurs could not be compared for lateral asymmetries as there were too few left-sided cases with bilateral responses. Of the right-sided cases $4 / 6$ had larger responses on the right hand. There was no consistent lateral asymmetry in skin temperature.

\section{Discussion}

A new light is cast on neurogenesis in migraine through evidence of extremes of autonomic responsiveness to sensory stimuli during the interictal phase, the direction of which was predictive of the side of the headache. Previously, extreme sensitivity or over-responsiveness, seen in the symptoms of phonophobia and photophobia, have been regarded as predispositional factors. ${ }^{9}$ Our results indicate that under-responsiveness is also relevant. The fact that both extremes of reactivity are implicated points to a deficiency in a mechanism that regulates responsiveness rather than inhibits responsiveness. The diffuse thalamic projection system which regulates regional patterns of cerebral activity may provide such a mechanism. It is implicated both in the mediation of the orienting response and in its rate of habituation with stimulus repetition. ${ }^{1516}$ The hypothesised role for autonomic dysregulation in migraine ${ }^{14}$ which drew on the symptom of nausea, a cardinal feature of migraine ${ }^{20}$ coupled with evidence of arhythmia in the EEG is substantiated by electrodermal activity which is sympathetic. A deficiency in regulating central activation may account for why states of relaxation and sleep are precipitants of migraine as well as states of stress and tension.

How does a deficiency in regulating central activation relate to the laterality of pain in migraine and lead to the specific relationships between left-sided pain and sympathetic under-responsiveness, and right-sided pain and over-responsiveness? Cerebral asymmetry has recently been associated with responsiveness and rate of habituation of electrodermal responses to sensory stimuli. Heilman et al ${ }^{18}$ found that patients with dysphasia and lesions of the left hemisphere exhibited slow habituation of electrodermal orienting responses to a variety of sensory stimuli, whereas patients with unilateral spatial neglect and lesions of the right hemisphere were underresponsive. From this it was inferred that the opposite polarity of influences would obtain in the intact brain. Bilateral recordings of electrodermal responses in normals ${ }^{19}$ showed that rate of habituation to auditory stimuli correlated with the lateral asymmetry in the tracing, such that fast habituation was associated with left hemispheric influences and slow habituation with a dominance of right hemispheric influences. Accordingly migraine would appear to represent a state 
of extreme hemispheric imbalance, and it is this that underpins the laterality of pain. Migraine has previously been described as a state of hemispheric overload. ${ }^{24}$ Our results suggest that while the migraineur may be predisposed to a state of hemispheric overload, this is because of a deficiency in a mechanism that regulates regional cerebral activation, rather than because of lateralised structural lesions which may have permanent consequences on hemispheric specialisation. ${ }^{25}$ Dysregulation because of a dynamic process is more in keeping with the episodic nature of migraine during the lifespan, and the fact that migraine may often be inconsistent in its laterality from one attack to another, may vary during an attack, and may show inconsistencies of laterality between the neurological signs of hemiparesis, hemianopia and dysphasia. ${ }^{26}$

It has been proposed that cerebral asymmetries in habituation may predispose the left hemisphere to focal attention and the right hemisphere to broad, vigilant attention. ${ }^{19}$ The EP asymmetries reported by Kennard et al $^{11}$ may be interpreted in the light of this hypothesis. They showed EP asymmetries only in leftsided migraineurs in the direction of augmented left occipital EPs occurring in middle latency components influenced by attentional processes. As viewing a rotating checkerboard will require focal attentional processes, and because these engage the left hemisphere, then this should tax the capabilities of the left-sided migraineurs as their state of lateral imbalance overactivates the left hemisphere.

The dissociation between tonic and phasic activation in the first experiment may have been due to medication, as there was no such association in the second experiment when the patients were drug-free. Nevertheless in both studies the patients were troubled by frequent migraine attacks, suggesting that medication in the first sample was ineffective. Accordingly the bipolarity in the phasic rather than the tonic responses in migraine may be the more important predispositional factor. Sharpless and Jasper ${ }^{15}$ were the first to differentiate clearly between mechanisms underlying the short lasting phasic response and the longer lasting tonic response. They led the way in showing that peripheral stimulation can influence the cortex in three ways: via the brainstem reticular formation, via the specific nuclei of the thalamus, and the diffuse thalamic projection system. The brainstem reticular formation is responsible for tonic activation which may last for minutes. It has a long latency, yet exhibits rapid but sustained habituation which may not permit dishabituation for hours or days. In contrast stimulation via the specific thalamic nuclei barely outlasts the stimulus, has a latency of one or two $\mathrm{ms}$, and in the visual system relays information concerning contrast borders, hence the procedure of pattern reversal. It is the third route via the diffuse thalamic projection system that involves orienting and habituation processes. This has a latency of about $25 \mathrm{~ms}$ (longer in the electrodermal system) and persists for 15 seconds after stimulus offset. This system is responsive to unpatterned flashes. Accordingly it would appear that it is the diffuse thalamic projection system, a system which regulates regional cerebral activation, that underlies the habituation differences that distinguish left from right-sided migraineurs.

The involvement of thalamo-cortical systems in migraine offers a new perspective on the neurogenesis of migraine. Other approaches to the neural hypothesis have focused on different cortical or sub-cortical mechanisms. Similarities have been drawn between migraine and epilepsy. It has been proposed that synapses in the cortex by virtue of axon collaterals have capacity for self-excitatory behaviour, ${ }^{27}$ and that sensory discomfort induced by certain patterns of stripes may arise from a breakdown in focal cortical inhibition. ${ }^{28}$ At a sub-cortical level theorists have implicated the hypothalamus, once in the sixties ${ }^{29}$ and again more recently, ${ }^{3}$ drawing on factors such as alterations in water balance, dietary precipitants and changes in other basic drive states that precipitate migraine. Connections between the hypothalamus and the brainstem have also been invoked ${ }^{29}$ and it is of interest that stimulation of the locus ceruleus in animals produces vaso-motor changes akin to those of migraine: vaso constriction in the intra-cerebral circulation and vaso-dilatation in the extra-cerebral circulation. ${ }^{30}$ By virtue of anatomy and function the thalamus is strategically placed to mediate between the hypothalamus, brainstem and cortex. ${ }^{31}$ In support of our thalamo-cortical hypothesis evidence does exist to show that pathology of the thalamus can produce the neurological symptoms of classical migraine. $^{32}$

\section{References}

1 Gowers WR. A Manual of Diseases of the Nervous System Vol 11. London: Churchill, 1888:789.

2 Dalessio DJ. Wolff's Headache and Other Head Pains. New York: Oxford University Press, 1972.

3 Blau JN. Migraine pathogenesis: the neural hypothesis re-examined. J Neurol Neurosurg Psychiatry 1984; 47:437-42.

4 Dalessio DJ. Is there a difference between classic and common migraine? Arch Neurol 1985;42:275-6.

5 Ziegler DK. The headache symptom. Arch Neurol 1985;42:273-4.

6 Sakai F, Meyer JS. Regional cerebral hemodynamics during migraine and cluster headache measured by the Xenon-133 inhalation method. Headache 1978; 18:122-32. 
7 Olesen J, Larsen B, Lauritzen M. Focal hyperoemia followed by spreading oligemia and impaired activation of rCBF in classic migraine. Ann Neurol 1981; 9:344-52.

8 Lauritzen M, Olesen J. Regional cerebral blood flow during migraine attacks by Xenon-133 inhalation and emission tomography. Brain 1984;107:447-61.

9 Selby G. Migraine and its Variants, Sydney: Adis Health Science Press, 1983.

10 Parsonage $M$. Electroencephalographic studies in migraine. In: Pearce J, ed. Modern Topics in Migraine. London: Heinman, 1975:72-84.

11 Kennard C, Gawel N, Rudolph N de M, Clifford-Rose F. Visual evoked potentials in migraine subjects. In: Res Clin Stud Headache. Basel: Karger, 1978;6:73-80.

12 Picton TV, Campbell KB, Baribeau-Braun J, Proux GB. The neurophysiology of human attention: A tutorial review. In: J Requin, ed. Attention and Performance, 7. Hillsdale: Erlbaum, 1978.

13 Connolly J, Gawel M, Clifford-Rose F. Migraine patients exhibit abnormalities in the visual evoked potential. J Neurol Neurosurg Psychiatry 1982; 45:464-7.

14 Jay GW. Epilepsy, migraine, and EEG abnormalities in children: A review and hypothesis. Headache 1982;22:110-4.

15 Sharpless $\mathrm{S}$, Jasper H. Habituation of the arousal reaction. Brain 1956;79:655-80.

16 Lynn R. Attention, Arousal, and the Orientation Reaction. London: Pergamon, 1966.

17 Pribram KH, McGuiness D. Arousal activation and effort in the control of attention. Psychol Rev 1975;82:116-49.

18 Heilman KM, Schwartz MD, Watson RT. Hypoarousal in patients with the neglect syndrome and emotional indifference. Neurology 1978;28:229-32.

19 Gruzelier JH, Eves FF, Connolly JF. Habituation and phasic reactivity in the electrodermal system: Reciprocal hemispheric influences. Physiol Psychol 1981;9:313-7.
20 The Ad Hoc Committee on Classification of Headache. Arch Neurol 1962;6:173.

21 Cattell RB, Sheier JH. Handbook for the IPAT Anxiety Scale Questionnaire (Self Analysis Form). Windsor: NFER, 1963.

22 Connolly JF, Gruzelier JH. Amplitude and latency changes in the visual evoked potential (VEP) to different stimulus intensities. Psychophysiology 1982;19:599-608.

23 Herron J. (Ed) Neuropsychology of Left-handedness. London, Academic Press, 1980.

24 Crisp AH. Laterality of migraine and reported affect: The relevance of a hypothesis concerning the nature of migraine. J Affect Dis 1981;3:71-5.

25 Crisp AH, Karmen J, Potamianos G. Bhat AV. Cerebral hemisphere function and laterality of migraine. Psychother Psychosom 1985;43:49-55.

26 Peatfield RC, Gawel MJ, Clifford-Rose F. Asymmetry of the aura and pain in migraine. $J$ Neurol Neurosurg Psychiatry 1981;41:846-8.

27 Crick F, Mitchison G. The function of dream sleep. Nature 1983:304:111-4.

28 Wilkins A, Nimmo-Smith I, Tait A, et al. A neurological basis for visual discomfort. Brain 1984;107:989-1017.

29 Herberg SJ. The hypothalamus and aminergic pathways in migraine. In: Pearce $\mathrm{J}$, ed. Modern Topics in Migraine. London: Heinemann, 1975:85-95.

30 Lance JW, Lambert GA, Godesby PJ, Duckworth JW. Brainstem influences on the cephalic circulation: Experimental data from cat and monkey of relevance to the mechanism of migraine. Headache 1983; 23:258-65.

31 Bruyn GW. Putative patho-mechanisms of migraine: A critical appraisal. J Drug Res 1980;10:35-43.

32 Pay RG. Conative regulation of cortical activity by the reticular formation, hypothalamus, and thalamus. Int $J$ Neuroscience 1980;10:233-53.

33 Waddington MM. Atlas of Cerebral Angiography with Anatomic Correlation. Boston, Little, Brown \& Co, 1974:432. 